\title{
Divergent Trajectories of Urban Development in 287 Chinese Cities
}

\begin{abstract}
The urbanization and motorization of Chinese cities follow divergent trajectories. However, how the diversity occurred, particularly within the small and medium cities, is understudied. Using panel data from 287 cities from 2001 to 2014 and a time-series clustering method, this study identified representative trajectories along which Chinese cities were urbanized and motorized. Urbanization was measured by scale, wealth, urban form, and infrastructure; motorization by automobile, taxi, bus numbers, and subway lines. Chinese cities were classified into four clusters: 23 Cluster-1 cities were the large cities with heavy rails; 41 Cluster-2 cities were the low-density wealthy cities with auto-oriented mobility; 134 Cluster-3 cities were the low-density medium-wealth cities with moderate mobility levels; and 89 Cluster-4 cities were the highdensity poor cities with lowest mobility levels. Comparing to the traditional three-tier structure, exclusively based on political tiers, the four-cluster structure respects the multi-dimensional nature of cities and reflects the essential diversities among the medium and small cities. While political tiers remain critical, other features including scale, density, infrastructure, and mobility patterns are also important: scale differentiates Cluster-1 from others; low density characterizes Clusters 2 and 3; heavy rail and auto-oriented mobility respectively identify Clusters 1 and 2 . We contribute to China's urban development literature by explicitly examining the temporal dimension, analyzing both urbanization and motorization, and incorporating all the medium and small cities in China. The distinct patterns of Clusters 2, 3, and 4 are evident, and the variation within them were as important as that between them and large cities.
\end{abstract}

Keywords: urban development trajectory, Chinese cities, urbanization, motorization, clustering Authors: Shenhao Wang, Jinhua Zhao 


\section{INTRODUCTION}

Chinese cities and their urbanization and motorization processes are highly diverse. Most prior studies emphasized the difference between the large cities and the rest or the regional disparities. The heterogeneity among the small and medium cities is less understood. Most prior studies used the cross-sectional data and could not examine the process of city development. Without the knowledge of temporal dynamics, it is difficult to inform cities on what actions to take in order to shape their future development trajectories. No studies to date examined simultaneously both urbanization and motorization processes of Chinese cities.

This study seeks to identify several representative trajectories along which Chinese cities were urbanized and motorized by using the panel data from 287 Chinese cities from 2001 to 2014. Chinese cities experienced their major urbanization and motorization from 2001 to 2014. We hypothesize that the cities follow different trajectories of development during those years, and their divergent trajectories can be summarized by several representative ones. We integrated our dataset from four groups of data sources: the China Premium Database from China Economic Information Center (CEIC), China City Yearbooks from 2001 to 2014, yearbooks at the municipal and provincial levels from 2001 to 2014, and the China Association of Metros. For each year-city pair (14 years * 287 cities) we measured urbanization along four dimensions: scale, wealth, urban form, and infrastructure, and motorization along four dimensions automobile, taxi, bus numbers, and subway lines. We applied a time-series clustering method, specifically Gaussian mixture model, to infer the representative trajectories of urban development.

The contribution of this study is three-fold. First, on the substantial knowledge of Chinese cities, we evidenced that there existed a considerable heterogeneity of urban development and identified four clusters of cities with representative trajectories of development. The heterogeneity is effectively captured by eight dimensions associated with the four clusters. Compared with the conventional three-tier city structure, the four-cluster structure better respects the multidimensional nature of urbanization and motorization and highlights the essential diversities among the medium and small cities. Second, we illustrated the use of the time-series clustering method in city analysis, which is able to portray the dynamic changes of cities, rather than only the static pictures. The third contribution is a major effort of data collection and synthesis. We integrated four sets of data sources, extracted eight variables for 287 Chinese cities from 2001 to 2014, and cross-validated the data by inspecting national, provincial, and city yearbooks. We hope that this dataset can support many future studies of Chinese cities.

The following sections will introduce the literature, city feature selection, data collection and synthesis, Gaussian mixture model and results, and discussions and limitations.

\section{LITERATURE REVIEW}

We will first review the studies focusing on city clustering and then those analyzing the urbanization and motorization of Chinese cities. Some literature that demonstrates feature selection and clustering methods will be discussed in the method section. 


\section{City Clustering}

It has been long and widely acknowledged that there exists significant heterogeneity across cities. Many scholars sought to classify cities using intuition or basic statistical tools decades ago (Hook \& Replogle, 1996; Neal, 2006; Nelson, 1955). Nelson (1955) classified American cities to match people's intuition: Detroit was categorized as a manufacturing city, while Rochester was categorized as a professional town. Hook and Replogle (1996) classified Asian cities based on motorization patterns and found four types of cities, including nonmotorized transport dominant cities, mixed traffic cities, public transportation dominated cities, and private motor vehicle dominated cities. Even without any advanced machine learning tools, scholars practiced city classification to reduce the large number of cities to some representative ones for comprehension.

Recently, with more mature clustering algorithms and more available data, scholars started to apply more rigorous methods to analyze the heterogeneity of cities through classification (Derudder, Witlox, \& Catalano, 2003; Frenkel, 2004; Priester, Kenworthy, \& Wulfhorst, 2013). By using the data of worldwide 234 cities, Derudder et al. (2003) used network analysis to reduce the cities to five-level hierarchies. By using 59 key transportation indicators and applying hierarchical clustering techniques, Priester et al. (2013) classified the worldwide cities into six categories, including hybrid cities, auto cities, transit cities, nonmotorized cities, paratransit cities, and traffic-saturated cities. By applying the hierarchical clustering algorithm to land use variables, Frenkel (2004) clustered the cities in Israel into two groups: heterogeneous and specialized land-use cities. These studies had distinct purposes, so they used different features. To analyze travel patterns, travel mode data were used (Priester et al., 2013); to analyze land use patterns, land use data were used (Frenkel, 2004). They used the clustering algorithms because clustering can reduce the considerable heterogeneity to several segments, each of which can be summarized by one representative entity. Usually the representative entities are named, such as transit cities or specialized land-use cities, to capture the essential characteristics of the clusters.

\section{Urbanization and Motorization of Chinese Cities}

Similarly in China, the cities have tremendous heterogeneity along both the temporal and the cross-sectional dimensions. Along the temporal dimension, China's urbanization has been growing at an extraordinary pace. About $27 \%$ of its population was urbanized in the 1990 s, while the number increased to 54\% in 2013 (Shen, Chen, \& Pan, 2016). The rapid urbanization was associated with some spatial suburbanization, which started as early as the 1980s (Zhou \& Meng, 1998). Between 2000 and 2014, the total number of passenger cars in China grew from 16.1 to 146.0 million, indicating a rapid motorization process (National Bureau of Statistics of China, 2015). The dramatic temporal changes imply that we should not ignore the temporal dimension in our clustering practice. Along the cross-sectional dimension, the heterogeneity of the urbanization and motorization of cities is also pronounced. Demurger (2001) analyzed how the economic growth varied with infrastructure investment across 24 provinces. Yi and Liu (2015) analyzed how the number of green employment opportunities and businesses varied with different energy policies across municipalities. Wu, Zhao, and Zhang (2016) explored how the number of automobiles was associated with GDP, population density, and other variables differently across 32 provincial capitals. In fact, the heterogeneity cross Chinese cities is rather intuitive: some major Chinese cities, such as Beijing and Shanghai, have become quite wealthy 
and well known over the world, while some remote Chinese cities are still struggling with poverty.

Acknowledging the heterogeneity, some studies sought to classify Chinese cities by using clustering algorithms. Guo, Wang, Shi, and Cao (2012) applied the DBSCAN algorithm to cluster the time-series housing price data, identifying six types of cities. Most germane to our analysis is Heikkila and $\mathrm{Xu}$ (2014), which applied a hierarchical clustering algorithm to cluster the policy contents of 286 Chinese cities. The cities were grouped into seven categories: land use planning, economic development, urban expansion, public management, urban-rural integration, public-private partnerships, and poverty alleviation clusters. Other studies mainly focused on either one single dimension or several dimensions of the Chinese cities in one particular region of China, without showing a panoramic view of all of the Chinese cities (Bradshaw \& Zhou, 1988; Yan \& Liu, 2009; Zhu \& Zhang, 2009). Moreover, the temporal dimension was not considered in the prior clustering analyses for Chinese cities.

The clustering method pertains to the commonsense tier structure of Chinese cities. While we did not find much academic literature about the tier structure, this topic could be found on many websites (Gardner, 2013; Mafengwo Net, 2017; Urban Legend Website, 2017). There is no consensus way to define the tier structure, although commonly used criteria include political tiers, GDP, and population (Cheng, 2017; Urban Legend Website, 2017). The formal political configuration is the three-tier structure: Tier 1, 4 cities directly controlled by the central government; Tier 2, 28 provincial capital cities; and Tier 3, about 250 prefectural cities. In this structure, the 250 cities are lump together even though they have considerable diversities in their urbanization and motorization levels and trajectories.

Overall, most existing studies clustered Chinese cities along one specific dimension (Guo et al., 2012; Heikkila \& Xu, 2014), using cross-sectional method (Heikkila \& Xu, 2014; Zhu \& Zhang, 2009) and heavily tilted towards the major cities.

\section{FEATURE SELECTION AND DATA COLLECTION}

\section{Feature Selection}

To operationalize the research, we identified four dimensions of the urbanization process: city scale, wealth, urban form, and infrastructure investment and chose one indicator for each of them: total urban population, GDP per capita, urban density, and road area per capita, respectively; we identified four dimensions of the motorization process and measured them by automobile ownership per capita, taxi per capita, bus number per capita, and subway length per capita.

The first two variables, total urban population and GDP per capita, have been included in nearly every study that uses cities as the unit of analysis (Dargay, Gately, \& Sommer, 2007; Glaeser, 1998; Ingram, 1998). The statistical association among wealth, scale, urban density, and road investment has been widely supported by many studies (Glaeser, 1998; Ingram, 1998; Rietveld, 1994; Zegras, 2003). The connections between auto ownership, buses, subway lines, urban density, and wealth have also been widely discussed, documented, and debated (BaumSnow \& Kahn, 2000; Cervero \& Landis, 1997; Crane, 1996; Gómez-Ibáñez, Tye, \& Winston, 2011; Newman \& Kenworthy, 1989; Pickrell, 1999; Waddell, 2011; Zhang, 2004).

While these eight dimensions and their indicators capture the main aspects of urbanization and motorization, we acknowledge that they are not intended to be comprehensive, 
and there can be debates on both the selection of the eight dimensions and the choice of the specific indicators for them. We consciously made important tradeoffs between conceptual needs, model sparsity, and data availability. Since our study covers a time span of 14 years and the complete 287 Chinese cities, every additional variable would make the data collection and validation much more challenging. In some instances, the ideal measures are simply missing or not consistent. Below are two examples: 1) we sought to measure motorization by mode shares in each city. However, a complete mode share database for all Chinese cities across 14 years does not exist. 2) We chose not to use urbanization rates even though it seemed an obvious choice, because there lacks of a consistent measurement of urbanization rates across cities and over the years. In addition to the data availability, we were cognizant of the model sparsity: we did not opt for many variables simply because we had them. Using too many features in clustering analysis has its drawbacks, and in particular it dilutes the interpretability of resulting clusters.

\section{Data Collection and Variable Definition}

Data was compiled and synthesized into one integrated database from four categories of data sources: 1) the China Premium Database from CEIC, 2) China City Yearbooks from 2001 to 2014, 3) yearbooks at the municipal and provincial levels from 2001 to 2014, and 4) the China Association of Metros. We used the CEIC database as the basis, and refined it by cross validating fourteen China City Yearbooks (2001-2014) and numerous municipal and provincial yearbooks. We examined the outliers in the CEIC database and replaced the incorrect values by referencing the corresponding data points in the specific yearbooks. The subway length was obtained from the website of the China Association of Metros.

Even after we confirmed the set of the eight indicators, the precise definition of the variables was not trivial. For example, population is the most basic variable and seems straightforward. However, due to the dichotomy of locals vs. migrants and agricultural vs. nonagricultural Hukou, there are at least five population variables to be considered: 1) census-based total population, 2) year-based total population, 3) registered population, 4) registered urban population, and 5) registered non-agricultural population. But none of the five variables serve our purpose well and we actually constructed our own indicator of "total urban population" by combining three of the five variables. We reported the detailed data processing of the eight variables in Appendix I. Finally we developed one integrated database of 287 cities by 14 years by 8 indicators.

\section{MODELING METHODS}

The premises of our study are that cities followed various urbanization and motorization trajectories and that their common threads can be identified by applying the clustering algorithm. Since the socio-economic and transportation conditions evolve over time, it is more relevant to cluster the time series variables than the static ones.

There are three major categories of time-series clustering methods (Liao (2005): rawdata-based, feature-based, and model-based. The raw-data-based clustering approaches use the raw data as the features of clustering, and the primary concern is how to pick the relevant dissimilarity measure. For instance, researchers may not use Euclidean and Mikowski distances, 
but those that more formally incorporate the information of temporal correlation, such as dynamic time warping distances, probability-based distances, and Kullback-Liebler distance. The feature-based approaches use the features extracted from the raw data for clustering. The method

of extracting features is often application dependent; thus, one crude way is to simply use the perceptually important points (Fu, Chung, $\mathrm{Ng}, \& \mathrm{Luk}, 2001)$, and a more rigorous way is to use a formal feature extraction method, such as Principal Component Analysis (PCA) (Shaw \& King, 1992). The model-based approaches use the features extracted from the models assumed for each cluster. The models are often the dynamic models, such as ARMA, AR, HMM, Markov Chain models, or even a simple Gaussian distribution, with features such as residuals, coefficients, parameters, or even P-values of the models (Baragona, 2001; Beran \& Mazzola, 1999; Piccolo, 1990). In fact, the model-based clustering methods are closely related to the latent class models used in the transportation field (Ben-Akiva et al., 2002; Walker \& Ben-Akiva, 2002).

It is difficult to assess the three time-series clustering approaches in a generic way. They all require the decisions on feature extraction, model specification, and dissimilarity measures. The choice of the clustering method depends on the specific question of interest and the characteristics of the data. This study followed a feature-based approach, using: 1) the mean value, the average growth (first difference), and the average curvature (second difference) of each variable as the clustering features; 2) The Gaussian mixture model (GMM) as the model specification; and 3) the Euclidean distance as the dissimilarity measure. The reasoning of the three choices is as follows. First, a feature-based approach can reduce the noise in the data and the three features capture key patterns of the development level and trajectory. For instance, we pay attention to the average wealth of a city and the average GDP growth, excluding the nuanced local yearly fluctuations. The mean value, first difference, and second difference are calculated based on the time series of each variable in each city. We opted to use the first difference (average absolute annual growth) rather than percentage growth rate in order to avoid artificially high growth rate of the variables with very low base. Second, we settled on the GMM, which is more flexible than the prevalent K-means method. In fact, if the Gaussian distributions of each cluster have $\sigma$ variances, then the GMM approaches the K-means algorithm which $\sigma$ goes to zero (Kulis \& Jordan, 2011). The GMM also provides more insight than the K-means approach: the GMM result includes the probability weighting matrix and the weights of each cluster represented by the variances. Third, the Euclidean distance is used as the dissimilarity measure since the three extracted features of each variable already reduced the temporal correlation in the initial time-series data. As illustrated by Liao (2005), there is a tradeoff between the feature extraction and the dissimilarity choice. Researchers either extract features to reduce the temporal correlation and then apply a conventional dissimilarity measure such as the Euclidean distance, or use raw data with a new dissimilarity measure such as the dynamic time warping distance. Therefore, we chose Euclidean distance after the reduction of the raw data to the three features.

\section{RESULTS}

\section{Descriptive Statistics}

Figure 1 plots the trajectories of the four urbanization measures, shown in the left panel, and the four motorization measures, shown in the right panel, for the 287 Chinese cities from 2001 to 2014. In the eight graphs, one curve represents one city's evolution throughout the 14 years. Six variables exhibited sustained growth, including total urban population, GDP per 
capita, road areas, auto per capita, bus per capita, and subway lengths, but the degrees of the growth varied by city and by measure. In contrast, the urban density had an overall downward trend, and the decline was dramatic in some cities. The taxi per capita, on average remained steady. These results are largely consistent with the intuition. During the fourteen years, Chinese cities experienced very rapid urbanization, economic growth, motorization, and public transit expansion.
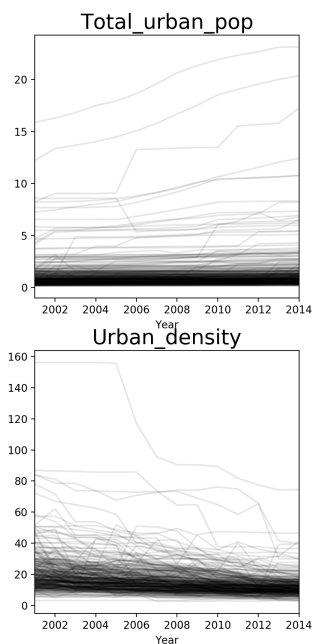
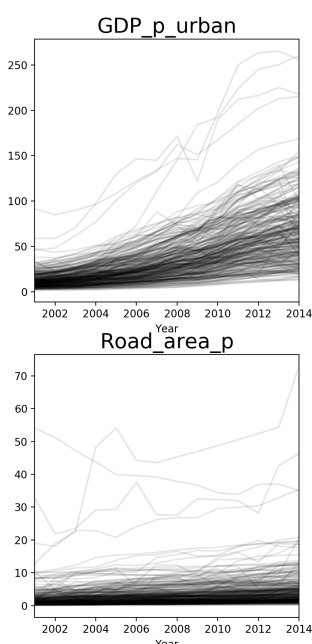

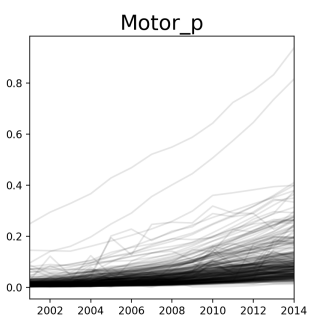

Bus_num_p

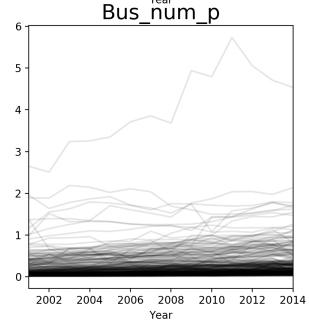

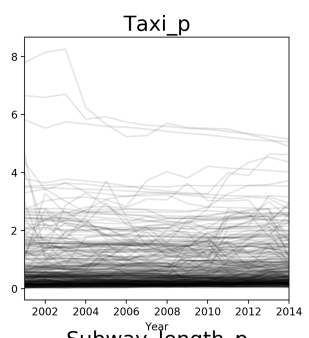

Subway_length_p

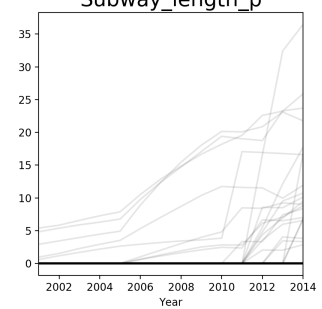

FIGURE 1 - Trajectories of 8 Variables of 287 Chinese Cities; Left: 4 Socioeconomic Variables; Right: 4 Transportation Variables

In addition to the overall growth, it is worth noting three patterns revealed in Figure 1. The first is the concurrent urbanization and suburbanization. The rise of total urban population describes urbanization at the regional level, while the sharp decrease of urban density indicates suburbanization at the metropolitan level, consistent with prior studies (Zhou \& Meng, 1998). The second is the declining urban density and increasing road area per capita, portraying a considerable urban expansion supported by heavy road investment. The third is the comparison between auto numbers and public transit, measured by the number of buses and length of subway lines. Auto growth was much faster than the growth of bus numbers, and was much more prevalent than that of the subway lines.

\section{Clustering Results}

We examined the multiple possibility of three, four, five and six clusters. After comparing the AIC and BIC scores and considering the interpretability of the clustering results, we opted for four clusters. For more detailed discussion about the selection of models and cluster numbers, please see Appendix II. Figure 2 plots the central trajectories of the four clusters of the eight variables. Table 1 summarizes the descriptive statistics of the four clusters, including three panels: Panel 1 shows the counts and population of cities; Panel 2 the mean comparison of the eight indicators, and their annual growth; and Panel 3, the exemplary cities in each cluster. 
Table 2 summarizes the key characteristics of the clusters.
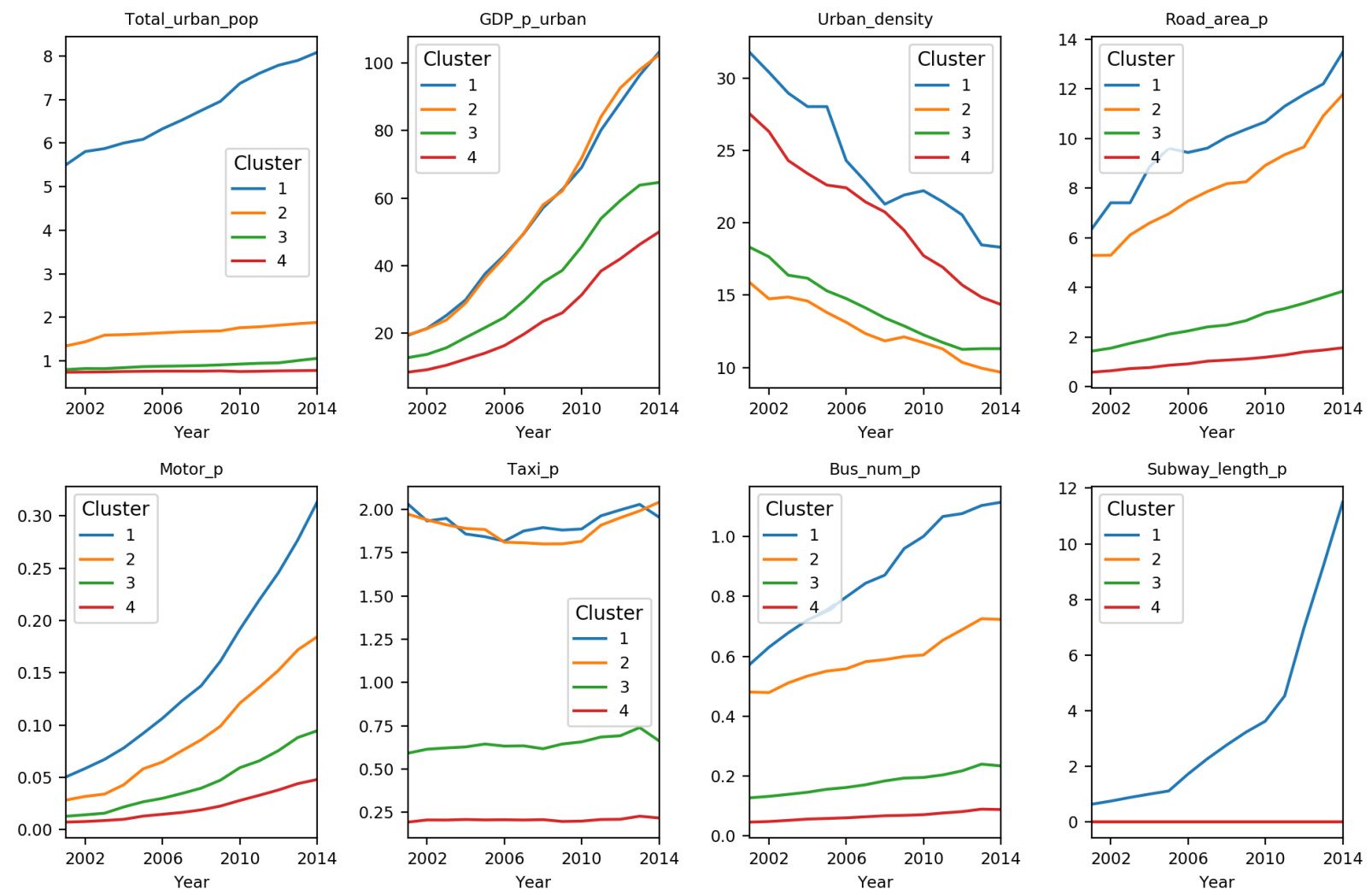

FIGURE 2 - Trajectories of Eight Indicators for Four Clusters; 1st Row: Population, GDP, Urban Density, Road Area; 2nd Row: Motor, Bus, Subway, Taxis

TABLE 1 Statistical Summaries of the Four Cluster Cities

\begin{tabular}{lcccc}
\hline & Cluster 1 & Cluster 2 & Cluster 3 & Cluster 4 \\
\hline Panel 1: Counts & & & & \\
\hline Counts & 23 & 41 & 134 & 89 \\
Counts (\%) & $8.0 \%$ & $14.3 \%$ & $46.7 \%$ & $31.0 \%$ \\
Population (Million) & 155.3 & 67.4 & 120.8 & 68.6 \\
Population (\%) & $37.7 \%$ & $16.3 \%$ & $29.3 \%$ & $16.6 \%$ \\
\hline Panel 2: Mean Comparison & & & & \\
\hline Total_urban_pop (Million) & 6.75 & 1.67 & 0.90 & 0.76 \\
GDP_p_urban (1000 Yuan/person) & 55.9 & 56.5 & 35.6 & 24.9 \\
Urban_density (Person/1000 sq meters) & 24.2 & 12.6 & 14.1 & 20.6 \\
Road_area_p(Sq meters/person) & 9.90 & 8.05 & 2.54 & 1.05 \\
Motor_p(Motor/1000 persons) & 151 & 92 & 45 & 22 \\
Taxi_p (Taxi/1000 persons) & 1.923 & 1.895 & 0.647 & 0.207 \\
Bus_num_p (Bus/1000 persons) & 0.871 & 0.591 & 0.178 & 0.066 \\
Subway_length_p (Meters/1,000 persons) & 3.59 & 0.000 & 0.000 & 0.000 \\
\hline
\end{tabular}




\begin{tabular}{|c|c|c|c|c|}
\hline FD_Total_urban_pop (Million) & 0.199 & 0.042 & 0.020 & 0.003 \\
\hline FD_GDP_p_urban (1000 Yuan/person) & 6.437 & 6.366 & 3.986 & 3.188 \\
\hline FD_Urban_density(Person/1000 sq meters) & -1.036 & -0.477 & -0.540 & -1.014 \\
\hline FD_Road_area_p (Sq meters/person) & 0.549 & 0.498 & 0.185 & 0.076 \\
\hline FD_Motor_p (Motor/1000 persons) & 20 & 12 & 6 & 3 \\
\hline FD_Taxi_p (Taxi/1000 persons) & -0.006 & 0.005 & 0.006 & 0.002 \\
\hline FD_Bus_num_p(Bus/1000 persons) & 0.042 & 0.019 & 0.008 & 0.003 \\
\hline FD_Subway_length_p (Meters/1000 persons) & 0.835 & 0.000 & 0.000 & 0.000 \\
\hline \multicolumn{5}{|c|}{ Notes: FD stands for first difference (average growth) } \\
\hline \multicolumn{5}{|c|}{ Panel 3: Selected Cities (Randomly Selected from Each Cluster) } \\
\hline \multirow{20}{*}{ Selected Cities } & Xian & Xining & Fushun & Bazhong \\
\hline & Tianjin & Panjin & Xiangtan & Zhangzhou \\
\hline & Shanghai & Weihai & Pingdingshan & Jian \\
\hline & Kunming & Hefei & Tongliao & Yichun \\
\hline & Nanjing & Changchun & Jining & Longnan \\
\hline & Guangzhou & Benxi & Dezhou & Leshan \\
\hline & Beijing & Shantou & Tieling & Baoshan \\
\hline & Chongqing & Daqing & Wulanchabu & Huaihua \\
\hline & Shenyang & Erdos & Xinyu & Fuzhou \\
\hline & Ningbo & Yichun & Nantong & Ankang \\
\hline & Suzhou & Yinchuan & Langfang & Shangrao \\
\hline & Wuxi & Huizhou & Yichang & Dingxi \\
\hline & Shenzhen & Hohhot & Cangzhou & Linfen \\
\hline & Foshan & Xiamen & Zhaoqing & Fuyang \\
\hline & Zhongshan & Zhoushan & Shaoxing & Putian \\
\hline & Zhengzhou & Karamay & Taizhou & Anshun \\
\hline & Wuhan & Jinchang & Huaibei & Nanchong \\
\hline & Suzhou & Lhasa & Binzhou & Qujing \\
\hline & Hangzhou & Taiyuan & Shuozhou & Mianyang \\
\hline & Chengdu & Huainan & Qitaihe & Tonghua \\
\hline
\end{tabular}


TABLE 2 Characteristics of the Four Clusters

Urbanization

\begin{tabular}{|c|c|c|c|c|}
\hline Clusters & $\begin{array}{c}\text { Scale } \\
\text { (Total Urban } \\
\text { Population) } \\
\end{array}$ & $\begin{array}{c}\text { Wealth } \\
\text { (GDP per } \\
\text { capita) }\end{array}$ & $\begin{array}{c}\text { Form } \\
\text { (Urban Density) }\end{array}$ & $\begin{array}{c}\text { Infrastructure } \\
\text { (Road Area } \\
\text { per capita) }\end{array}$ \\
\hline $\begin{array}{l}\text { Cluster 1: } \\
\text { Large cities with heavy rails }\end{array}$ & Large & Rich & High & $\begin{array}{c}\text { Heavy } \\
\text { investment }\end{array}$ \\
\hline $\begin{array}{l}\text { Cluster 2: } \\
\text { Low-density wealthy cities with } \\
\text { auto-oriented mobility pattern }\end{array}$ & Medium & Rich & Low & $\begin{array}{c}\text { Heavy } \\
\text { investment }\end{array}$ \\
\hline $\begin{array}{l}\text { Cluster 3: } \\
\text { Low-density medium-wealth cities } \\
\text { with moderate mobility }\end{array}$ & Small & Medium & Low & $\begin{array}{l}\text { Low } \\
\text { investment }\end{array}$ \\
\hline \multirow[t]{2}{*}{$\begin{array}{l}\text { Cluster 4: } \\
\text { High-density poor cities with lowest } \\
\text { mobility levels }\end{array}$} & Small & Poor & High & $\begin{array}{c}\text { Low } \\
\text { investment }\end{array}$ \\
\hline & \multicolumn{4}{|c|}{ Motorization } \\
\hline Clusters & Auto per capita & $\begin{array}{l}\text { Taxi per } \\
\text { capita }\end{array}$ & Bus per capita & $\begin{array}{l}\text { Subway lines } \\
\text { per capita }\end{array}$ \\
\hline $\begin{array}{l}\text { Cluster 1: } \\
\text { Large cities with heavy rails }\end{array}$ & High & High & High & High \\
\hline $\begin{array}{l}\text { Cluster 2: } \\
\text { Low-density wealthy cities with } \\
\text { auto-oriented mobility pattern }\end{array}$ & Medium High & High & Medium high & Does not exist \\
\hline $\begin{array}{l}\text { Cluster 3: } \\
\text { Low-density medium-wealth cities } \\
\text { with moderate mobility }\end{array}$ & Medium & Medium & Medium & Does not exist \\
\hline $\begin{array}{l}\text { Cluster 4: } \\
\text { High-density poor cities with lowest } \\
\text { mobility levels }\end{array}$ & Low & Low & Low & Does not exist \\
\hline
\end{tabular}

Notes: Bolded features are the most effective differentiating ones for each cluster

The 23 cities in Cluster 1 were the large cities with heavy rails. They were characterized by their large scale, heavy rails, and the most rapid growth of the two features. As shown by Figure 2, the population and subway lines of the Cluster 1 cities were much higher than all the other clusters. Specifically, the average population growth was 199,000 residents per year, and heavy rails existed in only Cluster 1 cities. While automobiles grew quite rapidly in Cluster 1 cities, the percent growth rate of heavy rails $23.2 \%\left(0.835 / 3.59^{1}\right)$ was about twice as fast as automobiles $13.2 \%(20 / 151)$. Within the public transit sector, the Cluster 1 cities focused more on subway lines rather than bus numbers. Moreover, bus numbers had a steady increase from 2001 to 2010, but its growth slowed down after 2010; on the contrary, the subway lengths had a similarly stead increase from 2001 to 2010, but a sharp rise occurred after 2010. Hence these Cluster 1 cities were dominated by heavy rails rather than automobiles or buses.

\footnotetext{
${ }^{1} 0.835$ is the first difference and 3.59 is the average length of heavy rail per person. Hence the percentage growth rate is $0.835 / 3.59=23.2 \%$.
} 
Beyond the two key differentiating features, the Cluster 1 cities had the highest values in almost all other dimensions. First, these Cluster 1 cities were the richest cities with rapid GDP growth during the period. Second, the cities had the highest level but rapid decline of urban density. The rapid expansion of urban areas was supported by heavy road investment during the fourteen years. Third, while the Cluster 1 cities mainly featured its heavy rails, these cities had the highest overall mobility levels - the largest number of buses, auto, and taxis per capita. Lastly, the rapid growth of scale, wealth, infrastructure, automobiles, and subway lines has not leveled off by 2014, implying that the major cities have not reached the saturation point of development and that the rapid growth is still underway.

The 41 cities in Cluster 2 were the low-density wealthy cities with auto-oriented mobility pattern. First, the Cluster 2 cities had large wealth, but had only medium scale without much population growth. Contrasting to Cluster 1 cities, which grew in both wealth and scale, Cluster 2 cities grew only in wealth, but in not scale. Second, the Cluster 2 cities had the lowest urban density, sprawling with heavy road investment. Third, related to the low density and heavy road investment, the Cluster 2 cities were characterized by the auto-oriented mobility pattern. The automobile numbers grew rapidly by $13 \%$ per year, and the taxi numbers were very high, roughly the same as the Cluster 1 cities. On the contrary, the growth of buses was moderate, and subway lines did not exist at all. The local governments seemed to favor road investments over public transit. In fact, this concurrent low urban density and heavy road investment was in a sharp contrast to all other three clusters.

The Cluster 2 cities had even slightly higher wealth than Cluster 1. Many Cluster 2 cities were either costal cities or own a considerable amount of natural resources: rich of oil, coal, and iron fields; however, their populations are much smaller than cluster 1 cities. For instance, Daqing and Karamay owned the two largest oil fields in China; Taiyuan and Erdos were well known for their rich coal resources; 12 cities in Cluster 2, such as Haikou and Qingdao, were costal cities.

The 134 cities in Cluster 3 were the low-density middle-income cities with moderate mobility levels. These cities were the most "common" one in China. They accounted for the majority of Chinese cities, and had moderate levels in almost all eight dimensions. The Cluster 3 cities could be seen as the baseline model for analyzing Chinese cities.

The 89 cities in Cluster 4 were the high-density poor cities with lowest mobility levels. Their most striking pattern was the contrast between poverty and high-density. They were the poorest cities with both the lowest levels and growth of GDP, while their urban densities were very high, close to the Cluster 1 cities. Associated with the poverty, the road investment had the lowest level and slowest growth. This pattern was exactly the opposite to Cluster 2, where urban areas spread out with low density and considerable infrastructure investment. This urban form pattern seemed to portray an image of the cities initially concentrated in the urban center and expanding outward without the sufficient infrastructure. In fact, the association between poverty, high urban density, and low infrastructure investment, seems to resemble some cities in other developing countries, such as India and Brazil. Regarding the mobility, the Cluster 4 cities had the lowest values in the four measures of motorization. Within the four variables, auto growth was faster than that of the number of buses and taxis, and there were no subway lines at all.

Figure 1 shows three general patterns of Chinese cities, including the considerable urban expansion supported by heavy road investment, the concurrent urbanization and suburbanization, and the tension between automobiles and public transit. After clustering, however, we observe that these patterns did not hold for all Chinese cities. The exceptions include: first, the 
infrastructure investments in the Cluster 4 cities were deficient. Second, even though there was a constant tension between auto and subway in the Cluster 1 cities, the Cluster 2 cities favored auto-mobility over public transit, and the Cluster 3 and 4 cities had overall low mobility levels. Third, the concurrent urbanization and suburbanization happened to all the Chinese cities but with varying degrees. There were rapid urbanization in cluster 1 cities, much less in clusters 2 and 3 cities, and close to zero in cluster 4 cities.

\section{DISCUSSION}

This study analyzed the heterogeneous trajectories of the urbanization and motorization of 287 Chinese cities from 2001 to 2014 based on a time-series clustering method. We collected and cross-validated eight variables in fourteen years of the 287 Chinese cities. Beyond the common patterns of rapid wealth growth, concurrent urbanization and suburbanization, urban expansion supported by road investment and rapid automobile growth, we identified four clusters, each of which had its distinct urbanization and motorization pathway. The 23 Cluster 1 cities were the large cities with heavy rails. The most useful differentiating features are scale and subway lines. The 42 Cluster 2 cities were the low-density wealthy cities with auto-oriented mobility pattern. The 134 Cluster 3 cities were the low-density medium-wealth cities with moderate mobility levels. Lastly, the 89 Cluster 4 cities were the high-density poor cities with lowest mobility levels.

Figure 3 compares our four-cluster structure to the conventional three-tier structure based on the political hierarchy. There are two major contrasts: first, the three-tier structure does not reflect the multi-dimensional characteristics of cities. In our 4 cluster structure, while political hierarchy remains significant, other features are also important. Scale differentiates Cluster 1 from others; urban density is effective in separating Clusters 2 and 4; infrastructure investment reveals the large difference between Clusters 1 and 2 and Clusters 3 and 4; heavy rail is the hallmark of Cluster 1; and auto-oriented mobility effectively distinguishes Cluster 2 from others. Notably, our Cluster 1 does not differentiate between the conventional Tier 1 and Tier 2 cities. $^{2}$. One possible interpretation is that at the current stage of urban development, what differentiates Tier 1 and Tier 2 cities has gone beyond the physical characteristics represented by the eight variables in our clustering method. Potentially, qualitative factors such as good governance and quality of life are more important in differentiating the tier-1 cities such as Beijing and Shanghai from others. Second, the third-tier cities in the conventional structure, including 253 cities, conceal the rich complexity in the majority of the Chinese cities. In contrast, Clusters 2, 3 and 4 in our 4-cluster structure differentiate the three different sub-categories, each with distinct trajectories of urbanization and motorization.

\footnotetext{
${ }^{2}$ Even in the 5 cluster and 6 cluster structure we tried during the clustering process, the Tier 1 and Tier 2 cities do not separate from each other.
} 




FIGURE 3 - Comparison of Conventional 3-Tier Structure and 4-Cluster Structure

Lastly our clustering approach explicitly assigns the probability of a city belonging to a particular cluster. Table 3 shows that most cities clearly belong to the assigned cluster with very high probabilities while some cities do not deterministically belong to one single cluster. For example, Lijiang and Xuancheng locate between Clusters 3 and 4; and Panzhihua between Clusters 2 and 3.

TABLE 3 Probabilities of Selected Cities Belonging to Different Clusters

\begin{tabular}{rlrrrr}
\hline Cluster & City & $\begin{array}{r}\text { Probability } \\
\text { of being in } \\
\text { Cluster 1 }\end{array}$ & $\begin{array}{c}\text { Probability } \\
\text { of being in } \\
\text { Cluster 2 }\end{array}$ & $\begin{array}{c}\text { Probability } \\
\text { of being in } \\
\text { Cluster 3 }\end{array}$ & $\begin{array}{c}\text { Probability } \\
\text { of being in } \\
\text { Cluster 4 }\end{array}$ \\
\hline 1 & Beijing & $100 \%$ & $0 \%$ & $0 \%$ & $0 \%$ \\
1 & Shanghai & $100 \%$ & $0 \%$ & $0 \%$ & $0 \%$ \\
1 & Harbin & $100 \%$ & $0 \%$ & $0 \%$ & $0 \%$ \\
1 & Chongqing & $100 \%$ & $0 \%$ & $0 \%$ & $0 \%$ \\
1 & Tianjin & $100 \%$ & $0 \%$ & $0 \%$ & $0 \%$ \\
1 & Chengdu & $100 \%$ & $0 \%$ & $0 \%$ & $0 \%$ \\
2 & Huainan & $0 \%$ & $100 \%$ & $0 \%$ & $0 \%$ \\
2 & Hefei & $0 \%$ & $100 \%$ & $0 \%$ & $0 \%$
\end{tabular}




\begin{tabular}{|c|c|c|c|c|c|}
\hline 2 & Zhoushan & $0 \%$ & $100 \%$ & $0 \%$ & $0 \%$ \\
\hline 3 & Baishan & $0 \%$ & $0 \%$ & $100 \%$ & $0 \%$ \\
\hline 3 & Ezhou & $0 \%$ & $0 \%$ & $100 \%$ & $0 \%$ \\
\hline 3 & Huanggang & $0 \%$ & $0 \%$ & $100 \%$ & $0 \%$ \\
\hline 4 & Heihe & $0 \%$ & $0 \%$ & $0 \%$ & $100 \%$ \\
\hline 4 & Xianyang & $0 \%$ & $0 \%$ & $0 \%$ & $100 \%$ \\
\hline 4 & Weinan & $0 \%$ & $0 \%$ & $0 \%$ & $100 \%$ \\
\hline 2 & Panjin & $0 \%$ & $99 \%$ & $1 \%$ & $0 \%$ \\
\hline 3 & Xiangyang & $0 \%$ & $1 \%$ & $99 \%$ & $0 \%$ \\
\hline 3 & Jiaozuo & $0 \%$ & $0 \%$ & $99 \%$ & $1 \%$ \\
\hline 3 & Puer & $0 \%$ & $0 \%$ & $99 \%$ & $1 \%$ \\
\hline 2 & Jinchang & $0 \%$ & $98 \%$ & $2 \%$ & $0 \%$ \\
\hline 2 & Tongling & $0 \%$ & $98 \%$ & $2 \%$ & $0 \%$ \\
\hline 3 & Shaoxing & $0 \%$ & $2 \%$ & $98 \%$ & $0 \%$ \\
\hline 3 & Taian & $0 \%$ & $0 \%$ & $96 \%$ & $4 \%$ \\
\hline 2 & Huizhou & $0 \%$ & $95 \%$ & $5 \%$ & $0 \%$ \\
\hline 3 & Wuhu & $0 \%$ & $5 \%$ & $95 \%$ & $0 \%$ \\
\hline 4 & Puyang & $0 \%$ & $0 \%$ & $7 \%$ & $93 \%$ \\
\hline 4 & Jiujiang & $0 \%$ & $0 \%$ & $7 \%$ & $93 \%$ \\
\hline 3 & Xuchang & $0 \%$ & $0 \%$ & $90 \%$ & $10 \%$ \\
\hline 3 & Maanshan & $0 \%$ & $16 \%$ & $84 \%$ & $0 \%$ \\
\hline 3 & Fushun & $0 \%$ & $19 \%$ & $81 \%$ & $0 \%$ \\
\hline 3 & Panzhihua & $0 \%$ & $44 \%$ & $56 \%$ & $0 \%$ \\
\hline 3 & Lijiang & $0 \%$ & $0 \%$ & $53 \%$ & $47 \%$ \\
\hline 4 & Xuancheng & $0 \%$ & $0 \%$ & $48 \%$ & $52 \%$ \\
\hline
\end{tabular}

Overall, this study confirmed that the heterogeneous Chinese cities can be segmented into four representative trajectories of their development - not merely their state at a particular point in time. While Cluster 1 cities constitute the spotlight image of the rapidly developing Chinese cities, there are only 23 of them, accounting for less than $10 \%$ of the cities and less than $40 \%$ of the urban population. On the other hand, the large majority of the Chinese cities in Cluster 2, 3, and 4 have their unique characteristics. The differences among them have a multi-dimensional nature, and the key features vary for each cluster.

This study has its limitations, which point to future research opportunities. We reached our findings based on a large-scale data analysis without grounding it in the local context of the cities in each cluster. Future studies should validate our conclusion by conducting in-depth analysis into some representative cities. The clustering method in this study does not allow cities to change their cluster members, while admittedly some cities could change their membership during the 14 years period. Advanced methods (Campbell et al, 2013) can be used to allow dynamic cluster membership. 


\section{REFERENCES}

Baragona, R. (2001). A simulation study on clustering time series with metaheuristic methods. Quaderni di Statistica, 3, 1-26.

Baum-Snow, N., \& Kahn, M. E. (2000). The effects of new public projects to expand urban rail transit. Journal of public economics, 77(2), 241-263.

Ben-Akiva, M., McFadden, D., Train, K., Walker, J., Bhat, C., Bierlaire, M., . . Bunch, D. S. (2002). Hybrid choice models: progress and challenges. Marketing Letters, 13(3), 163175.

Beran, J., \& Mazzola, G. (1999). Visualizing the relationship between two time series by hierarchical smoothing models. Journal of Computational and Graphical Statistics, 8(2), 213-238.

Bradshaw, R., \& Zhou, Y. (1988). The classification of industrial function of Chinese cities, theory, method and results. Acta Geographica Sinica, 287-298.

Campbell, T., Liu, M., Kulis, B., How, J. P., \& Carin, L. (2013). Dynamic clustering via asymptotics of the dependent Dirichlet process mixture. Paper presented at the Advances in Neural Information Processing Systems.

Cervero, R., \& Landis, J. (1997). Twenty years of the Bay Area Rapid Transit system: Land use and development impacts. Transportation Research Part A: Policy and Practice, 31(4), 309-333.

Cheng, C. (2017). All you need to know about China city tiers. Retrieved from http://www.sinostep.com/all-you-need-to-know-about-china-city-tiers/

Crane, R. (1996). On form versus function: Will the new urbanism reduce traffic, or increase it? Journal of Planning Education and Research, 15(2), 117-126.

Dargay, J., Gately, D., \& Sommer, M. (2007). Vehicle ownership and income growth, worldwide: 1960-2030. The Energy Journal, 143-170.

Demurger, S. (2001). Infrastructure development and economic growth: an explanation for regional disparities in China? Journal of Comparative economics, 29(1), 95-117.

Derudder, Witlox, \& Catalano. (2003). Hierarchical tendencies and regional patterns in the world city network: a global urban analysis of 234 cities. Regional Studies, 37(9), 875-886.

Frenkel, A. (2004). Land-use patterns in the classification of cities: the Israeli case. Environment and Planning B: Planning and Design, 31(5), 711-730.

Fu, T.-c., Chung, F.-1., Ng, V., \& Luk, R. (2001). Pattern discovery from stock time series using self-organizing maps. Paper presented at the Workshop Notes of KDD2001 Workshop on Temporal Data Mining.

Gardner, R. (2013). China city tier system: how it works and why it is useful. Retrieved from http://nexus-pacific.com/blog/2013/7/9/china-city-tier-system-how-it-works-and-why-its$\underline{\text { useful }}$

Glaeser, E. L. (1998). Are cities dying? The journal of economic perspectives, 12(2), 139-160.

Gómez-Ibáñez, J. A., Tye, W. B., \& Winston, C. (2011). Essays in transportation economics and policy: a handbook in honor of John R. Meyer: Brookings Institution Press.

Guo, K., Wang, J., Shi, G., \& Cao, X. (2012). Cluster analysis on city real estate market of China: based on a new integrated method for time series clustering. Procedia Computer Science, 9, 1299-1305.

Heikkila, E., \& Xu, Y. (2014). Seven prototypical Chinese cities. Urban Studies, 51(4), 827-847.

Hook, W., \& Replogle, M. (1996). Motorization and non-motorized transport in Asia: Transport system evolution in China, Japan and Indonesia. Land Use Policy, 13(1), 69-84. 
Ingram, G. K. (1998). Patterns of metropolitan development: what have we learned? Urban Studies, 35(7), 1019-1035.

Kulis, B., \& Jordan, M. I. (2011). Revisiting k-means: New algorithms via Bayesian nonparametrics. arXiv preprint arXiv:1111.0352.

Liao, T. W. (2005). Clustering of time series data—a survey. Pattern recognition, 38(11), 18571874.

Mafengwo Net. (2017). Five tiers of Chinese cities. Retrieved from http://www.mafengwo.cn/travel-news/542672.html

National Bureau of Statistics of China. (2015). China Statistical Yearbook 2015: China Statistics Press.

Neal, Z. P. (2006). Culinary deserts, gastronomic oases: a classification of US cities. Urban Studies, 43(1), 1-21.

Nelson, H. J. (1955). A service classification of American cities. Economic geography, 31(3), 189-210.

Newman, P. W., \& Kenworthy, J. R. (1989). Gasoline consumption and cities: a comparison of US cities with a global survey. Journal of the American Planning Association, 55(1), 2437.

Piccolo, D. (1990). A distance measure for classifying ARIMA models. Journal of Time Series Analysis, 11(2), 153-164.

Pickrell, D. (1999). Transportation and land use.

Priester, R., Kenworthy, J., \& Wulfhorst, G. (2013). The diversity of megacities worldwide: Challenges for the future of mobility Megacity Mobility Culture (pp. 23-54): Springer.

Rietveld, P. (1994). Spatial economic impacts of transport infrastructure supply. Transportation Research Part A: Policy and Practice, 28(4), 329-341.

Shaw, C., \& King, G. (1992). Using cluster analysis to classify time series. Physica D: Nonlinear Phenomena, 58(1-4), 288-298.

Shen, Q., Chen, P., \& Pan, H. (2016). Factors affecting car ownership and mode choice in rail transit-supported suburbs of a large Chinese city. Transportation Research Part A: Policy and Practice, 94, 31-44.

Urban Legend Website. (2017). China's tiered city system explained. Retrieved from http://multimedia.scmp.com/2016/cities/

Waddell, P. (2011). Integrated land use and transportation planning and modelling: addressing challenges in research and practice. Transport Reviews, 31(2), 209-229.

Walker, J., \& Ben-Akiva, M. (2002). Generalized random utility model. Mathematical Social Sciences, 43(3), 303-343.

$\mathrm{Wu}, \mathrm{N}$., Zhao, S., \& Zhang, Q. (2016). A study on the determinants of private car ownership in China: Findings from the panel data. Transportation Research Part A: Policy and Practice, 85, 186-195.

Yan, W., \& Liu, J. (2009). Research on the theory and method of classifying and adjusting urban function: a case of Henan Province. Journal of Henan University, 265-270.

Yi, H., \& Liu, Y. (2015). Green economy in China: Regional variations and policy drivers. Global Environmental Change, 31, 11-19.

Zegras, C. (2003). Financing transport infrastructure in developing country cities: evaluation of and lessons from nascent use of impact fees in Santiago de Chile. Transportation Research Record: Journal of the Transportation Research Board(1839), 81-88. 
Zhang, M. (2004). The role of land use in travel mode choice: evidence from Boston and Hong Kong. Journal of the American Planning Association, 70(3), 344-360.

Zhou, Y., \& Meng, C. (1998). The Tendency of Subrubanization of Big Cities in China [J]. Urban Planning Review, 3, 004.

Zhu, X., \& Zhang, W. (2009). Guangdong Province city synthesis classification research. Journal of Huizhou University, 55-61.

\section{Appendices I and II}

Due to the word limit, they are not included in the main manuscript but available upon request. But we are attaching them as Supplemental Material for the review process.

\section{APPENDIX I DATA COLLECTION AND PROCESSING}

The choice to study a 14-year time span is partially due to the data availability of the China Economic Information Center (CEIC) database, which did not provide much information for the years before 2001 and has not updated the data later since 2015. It is also due to the fact that this time span characterizes the most relevant motorization and development period of Chinese cities: before 2001, most of Chinese cities had very few private automobiles. From 2001 to 2014, the number of the cities changed slightly. Four new cities were established: Bijie and Tongren in Guizhou Province in 2011; Sansha in Hainan Province in 2012; and Haidong in Qinhai Province in 2013. One city, Chaohu in Anhui province, was removed in 2011. Two cities, Xiangfan and Simao, changed their names to Xiangyang and Pu'er around 2010. Hence the total city number in 2014, as shown in the China City Yearbook, was 290, while the number was 287 in 2001 . We included only the 287 cities that have the complete records from 2001 to 2014 in our study.

While the four major data sources supplied numerous variables, we extracted eight indicators that were most germane to our analysis and most important according to prior studies (Baum-Snow \& Kahn, 2000; Cervero \& Landis, 1997; Crane, 1996; Dargay et al., 2007; Glaeser, 1998; Gómez-Ibáñez et al., 2011; Newman \& Kenworthy, 1989; Pickrell, 1999; Waddell, 2011; Zhang, 2004). These eight variables, although commonplace in urban and transportation analysis, are not easy to be obtained in a consistent fashion because of the data quality. More vexing is the different terminologies adopted in various statistical books for measuring the concepts. The detailed process of clarifying each variable is documented below.

\section{Population (Total urban population)}

This study used total urban population as the population indicator, which manifests the scale of a city. Although used prevalently, the precise definition of population is complicated due to the dichotomy of locals vs. migrants and agricultural vs. non-agricultural population in China. At least five population variables are often used, including 1) census-based total population, 2) year-based total population, 3) registered population, 4) registered urban population, and 5) registered non-agricultural population. However, none of the five variables serve our purpose perfectly. The census-based total population is not appropriate because the national government conducted a census only once every ten years. The year-based total population, provided by the CEIC database, is infeasible because it has on average 80 missing data points for each year. This 
is unsurprising because the statistics of migrants is particularly difficult to acquire. Registered population, registered urban population, and registered non-agricultural population are not adequate because any registered population variables exclude the migrants, which account for a sizeable portion of the total population, especially in major cities such as Beijing and Shenzhen. The ideal measure is the total urban population, which includes both locals and migrants, but excludes the residents living in the rural area.

To construct a better proxy for total urban population, we combined the information from year-based total population, registered population, and registered urban population and used the following formula:

Total urban population $=$ Registered urban population $* \frac{\text { Year based total population }}{\text { Registered population }}$

The underlying assumption is that the ratio of the total to the registered population is the same between the urban area and the region as a whole. This assumption is clearly not perfect, but the constructed proxy variable is more fitting than any of the five original variables and strikes a balance between feasibility and relevance.

\section{GDP per capita (Urban GDP / Total urban population)}

Associated with the population variable, we used urban GDP rather than total regional GDP to measure the economic status of a city. The urban GDP variable is collected from the 14 China City Yearbooks. This variable is of high quality because it contains nearly no missing data points from 2001 to 2014 . The GDP per capita variable is constructed as the ratio of urban GDP to total urban population.

\section{Urban density (Total urban population / Urban built area)}

The urban density variable is constructed as the ratio of total urban population to the built area. China City Yearbooks include three area variables (built areas, administrative areas, and urban areas) that measure the size of cities; and we opted for the built area. The built area reflects the real scale of urban development. The administrative area is inappropriate because this variable never changed except for the rare cases when new cities were established or old cities were removed even though the Chinese cities had grown significantly in the 14 years. While seemingly relevant, the urban area does not pertain to our study either because it only changed when the central government commanded that the central urban district incorporated adjoining rural districts. When this happened, the changes of urban areas were sudden and dramatic, rarely reflecting the incremental development patterns of the cities.

\section{Road area per capita (Total road area / Total urban population)}

The road area variable was collected from the CEIC database and cross-validated by inspecting relevant yearbooks. Thirteen errors were found in the CEIC database. Among the thirteen data points, the CEIC and the yearbooks shared twelve consistently. We removed these data points and replaced them by linear imputation. For instance, both the CEIC database and the yearbooks show that Ankang city in Shannxi province had the road area of $3.48 \mathrm{~km}$ squared in 2005, $0.93 \mathrm{~km}$ squared in 2006 and $4.50 \mathrm{~km}$ squared in 2007. The data point in 2006 was a clear 
mistake. We removed these data points and replaced them by linear imputation. The final road area per capita is constructed as the ratio of road area to total urban population.

\section{Automobile per capita (Automobile / Total registered population)}

This variable is most pertinent to our study, but also the most challenging to be collected, partially due to the convoluted terminology of all kinds of vehicles and also due to the varying data availability in yearbooks. As to the terminology, a provincial yearbook reports the number of total motor vehicles, which include five major categories: total automobiles, motorcycles, tractors, trailers, and others. Among the five categories, the total automobiles and motorcycles account for $90 \%$ of the total vehicle number. Within the category of automobiles, there are three types: passenger automobiles, freight automobiles, and others. The passenger automobiles and the freight automobiles are further segmented into three sizes: large, medium, and small. The other automobiles include three-wheel and four-wheel slow-speed automobiles. Although prevalently used, this terminology is not consistent across all the provinces. Some provinces only reported their total motor vehicles without further details. In about one third of the provincial yearbooks, no motor vehicle information at the city level was provided. In this case, we delved into the city level yearbooks for 14 years to collect motor information, increasing the effort significantly.

The validation of the motor variable is much more complex than the other variables. This is because the motor variable compiled by the CEIC is a mix of the total motor vehicle and the total automobile number, and this is a mistake in the CEIC database, probably due to the inconsistency of the terminology across city yearbooks. We cross-validated and corrected the CEIC dataset by identifying the illogical data points and replacing them with the information from provincial or city yearbooks. In total, we removed thirty-one outliers in the CEIC database after inspecting tens of corresponding city yearbooks. We substituted the data points of three provinces ${ }^{3}$ in the CEIC database after scanning over 42 provincial yearbooks. The motor indicator in our final database represents the number of automobiles in one city each year. Our revision clearly improved the data accuracy but we admit that some mistakes may still exist.

Please note that we used total registered population as the denominator instead of total urban population because the automobile numbers reported in the statistical yearbooks refer to the automobiles owned by the total registered population. The same is true for bus number per capita and taxi number per capita.

\section{Bus per capita (Bus numbers / Total registered population)}

Similar to the road area variable, the variable measuring the number of buses is included in the CEIC database and the China City Yearbooks, and the collection and validation are relatively easy. After validating the CEIC database, twelve data points were removed due to illogical fluctuations, and five cities' bus numbers were replaced by using the data from the China City Yearbooks.

\footnotetext{
${ }^{3}$ Yunnan, Hunan, and Shanghai. They were replaced due to the data error in CEIC
} 


\section{Taxi per capita (Taxi numbers / Total registered population)}

Similar to the bus variable, twelve wrong data points of taxi numbers in the CEIC database were removed and four cities' taxi numbers were substituted with the information from the China City Yearbooks.

\section{Subway length per capita (Subway line length / Total urban population)}

This variable was collected from the website of China Association of Metros.

\section{APPENDIX II SELECTING MODEL TYPES AND NUMBER OF CLUSTERS}

Two questions remain after we chose to use the Gaussian mixture model: how flexible the Gaussian distribution variance matrix should be and how many clusters we should use. To answer these two questions, we compared the AIC and BIC scores of the models, which vary by the flexibilities of the Gaussian distributions and the number of clusters, as shown in Figure 4. Gaussian distribution could be flexible to different degrees as it takes the form of a diagonal, spherical, or full variance matrix. As shown, the models with spherical variance matrix are inferior to the other two types due to the consistently high AIC and BIC scores. The comparison between the models with a full variance matrix and those with a diagonal variance matrix is not consistent between AIC and BIC. In terms of the AIC scores, the models with the full variance matrix perform slightly better than those with the diagonal variance, while they do significantly worse in terms of the BIC scores. ${ }^{4}$ To maintain a relatively parsimonious model and a simple computation process, we opted for the model with the diagonal variance matrix for our clustering analysis.

As for the cluster numbers, Figure 4 cannot provide conclusive results. The AIC and BIC scores of the models with the diagonal variance matrix decline very modestly when the number of clusters is larger than four. The statistical results do not suggest any superior cluster number, and the choice of the cluster number depends on the research objective and result interpretability. We examined the results of five and six clusters, but they did not reveal more interesting results than four clusters. Since a larger number of clusters reduce the interpretability of the result, we decide to set on four clusters.

After choosing four as the number of clusters, we ran the estimation hundreds of times to identify a robust clustering result with the minimum AIC and BIC. This is because the EM algorithm can only identify a local optimum and many rounds of searching are necessary to approximate the global optimum. There are caveats about the method we used. The clustering method and the $\mathrm{EM}^{5}$ algorithm do not guarantee a completely stable result. In fact, even after a large number of repeated estimations, one or two cities could change their cluster membership. Within the time-series clustering framework, we did not exhaust the potential of the method. For instance, with the clustering result, researchers could readily analyze the associations between automobile growth and socio-economic factors, and how they differ across the four clusters. Some simple linear transformations based on our Gaussian mixture model could help answer this question.

\footnotetext{
${ }^{4}$ This discrepancy is because BIC scores penalizes the complexity of models much more than AIC

${ }^{5}$ Expectation-maximization algorithm is commonly used in the models with latent variables. In our study, the latent variables are clusters.
} 

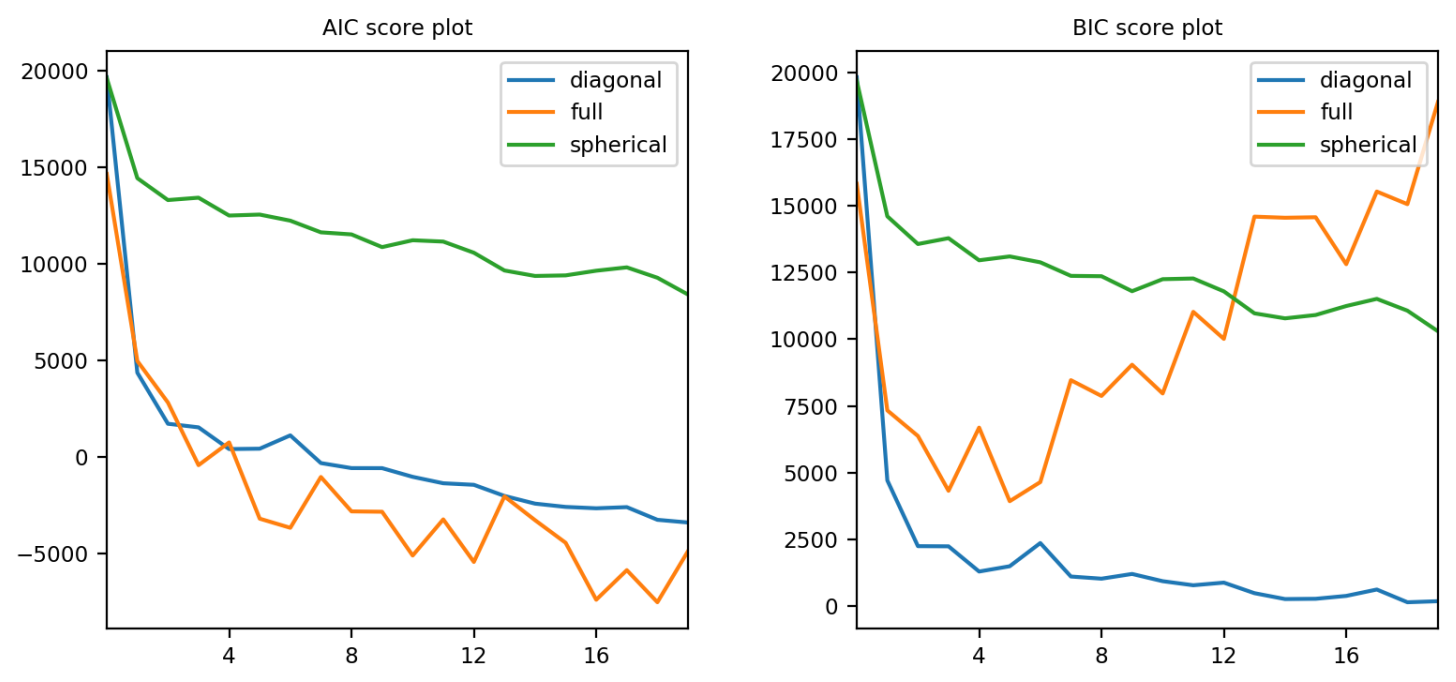

FIGURE 4 - AIC and BIC Scores of Clustering Models (K=2 to 20) 